\title{
Second Cancer Risk in Childhood Cancer Survivors Treated With Intensity-Modulated Radiation Therapy (IMRT): An Updated Analysis of More Than 10 Years of Follow-Up
}

Kathryn Tringale ${ }^{1}$, Dana Casey ${ }^{2}$, Gregory Niyazov ${ }^{1}$, Jessica Lavery ${ }^{1}$, Chaya Moskowitz ${ }^{1}$, Danielle Novetsky Friedman ${ }^{3}$, and Suzanne Wolden ${ }^{1}$

${ }^{1}$ Memorial Sloan Kettering Cancer Center

${ }^{2}$ University of North Carolina System

${ }^{3}$ Memorial Sloan Kettering

December 24, 2021

\author{
Abstract \\ radiation treatment (e.g., proton therapy). \\ (IMRT): An Updated Analysis of More Than 10 Years of Follow-Up \\ N. Friedman ${ }^{5}$, Suzanne L. Wolden ${ }^{1}$ \\ tion Oncology, University of North Carolina ${ }^{2}$ \\ Corresponding Author: \\ Kathryn R. Tringale, MD MAS \\ tringalk@mskcc.org \\ Department of Radiation Oncology, Memorial Sloan Kettering Cancer Center \\ 1275 York Avenue, New York NY 10065 \\ Word Count
}

Background It is unclear how intensity-modulated radiation therapy (IMRT) impacts long-term risk of second malignant neoplasms (SMNs) in childhood cancer patients. Procedure Patients aged 10 years, many solid SMNs after IMRT in childhood cancer survivors develop in the high dose region. These data serve as a foundation for comparison with other modalities of

Second Cancer Risk in Childhood Cancer Survivors Treated With Intensity-Modulated Radiation Therapy

Kathryn R. Tringale ${ }^{1}$, Dana L. Casey ${ }^{2}$, Gregory Niyazov ${ }^{3}$, Jessica A. Lavery ${ }^{4}$, Chaya Moskowitz ${ }^{4}$, Danielle

Department of Radiation Oncology ${ }^{1}$, Department of Medical Physics ${ }^{3}$, Department of Epidemiology and Biostatistics $^{4}$, Department of Pediatrics ${ }^{5}$, Memorial Sloan Kettering Cancer Center; Department of Radia-

Abstract: 250

Manuscript: 2547

Tables: 3

Figures: 3

Supporting Documents: None 
Short Running Title: Second Cancer Risk After IMRT

Keywords: Pediatrics, radiotherapy, second malignancies, survivorship, IMRT

A version of this publication was presented at the annual American Society of Radiation Oncology (ASTRO) meeting in Chicago, Illinois in October 2021. The abstract is available online: https://www.redjournal.org/article/S0360-3016(21)01523-6/fulltext.

Funding: Support for this work was provided to Memorial Sloan Kettering Cancer Center by a core grant from the National Cancer Institute (P30 CA008748).

\begin{tabular}{ll}
\hline Abbreviation & Phrase \\
\hline RT & Radiotherapy \\
IMRT & Intensity-modulated radiotherapy \\
IDL & Isodose level \\
CSI & Craniospinal irradiation \\
SIR & Standardized incidence ratio \\
EAR & Excess absolute risk \\
SMN & Second malignant neoplasm \\
MDS & Myelodysplastic syndrome \\
AML & Acute myeloid leukemia \\
PA & Posteroanterior \\
CNS & Central nervous system \\
\hline
\end{tabular}

\section{ABSTRACT}

Background

It is unclear how intensity-modulated radiation therapy (IMRT) impacts long-term risk of second malignant neoplasms (SMNs) in childhood cancer patients.

Procedure

Patients aged [?]21 years treated with IMRT between 1998-2009 and who survived [?]5 years after IMRT were included. SMN site in relation to isodose level (IDL) of IMRT was evaluated. Standardized incidence ratios (SIR) and excess absolute risks (EAR) were calculated. Cumulative incidences were estimated with death as a competing risk.

Results

Three hundred and twenty-five patients were included with median follow-up of 11.2 years from IMRT (interquartile range, 9.4, 14.0) among patients alive at the end of follow-up. Two hundred (62\%) patients had [?]10 years of follow-up and $284(87 \%)$ patients were alive at the time of analysis. Fifteen patients developed SMNs (11 solid, 4 hematologic). Median time from IMRT to solid SMN was 11.0 years (range, 6.8-19.2) with 10- and 15-year cumulative incidences $1.8 \%$ (95\% CI $0.7-3.9)$ and $3.5 \%$ (95\% CI 1.4-7.5), respectively; SIR was 13.7 (95\% CI 6.9-24.6) and EAR was 2.8 per 1,000 person-years (95\% CI 1.0-4.6). Eight solid SMNs developed within the IMRT field (100\% IDL [n=5], 80\% IDL [n=1], 50\% IDL [n=1], 40\% IDL $[n=1]$ ), one within the $70-80 \%$ IDL of a conventional field, one was out-of-field, and one could not be determined.

Conclusions

With median follow-up of $>10$ years, many solid SMNs after IMRT in childhood cancer survivors develop in the high dose region. These data serve as a foundation for comparison with other modalities of radiation treatment (e.g., proton therapy). 


\section{INTRODUCTION}

As treatments improve and the cohort of survivors from childhood cancer increases, the risk of treatmentrelated toxicity becomes even more relevant ${ }^{1}$. It is known that radiotherapy is an important risk factor in the development of second solid tumors in this radiosensitive pediatric population ${ }^{2-4}$. Intensity-modulated radiotherapy (IMRT) is a highly conformal technique that reduces the normal tissue volume exposed to a high radiation dose yet can increase the volume of normal tissue exposed to lower doses, as well as the total monitor units and integral dose to the patient ${ }^{5,6}$. Since radiation-induced carcinogenesis is thought to follow a no-threshold, linear model, it has been theorized that IMRT could increase the risk of SMN given the larger volume of normal tissue exposed to some radiation. To investigate this, a preliminary study of second malignancies in 242 childhood cancer survivors after IMRT at our institution was published in $2015^{7}$. At a median follow-up of 7.9 years, 4 patients developed a second solid cancer with a 10-year cumulative incidence of $3.3 \%$. Here, we seek to expand upon this analysis with a larger cohort and a median follow-up of more than 10 years.

\section{METHODS}

\section{Patients}

This study was a retrospective analysis of patients treated with IMRT at [?]21 years of age at Memorial Sloan Kettering Cancer Center (MSKCC) between December 1998 and February 2009. Patients were included if they had survived and had follow-up data for at least 5 years after IMRT initiation $(\mathrm{n}=325)$. The study was approved by the MSKCC Institutional Review Board/Privacy Board. Data collected included age at diagnosis and at IMRT initiation, sex, race, known hereditary cancer syndrome (e.g. , Li-Fraumeni, neurofibromatosis, or hereditary retinoblastoma), primary tumor histology and location, chemotherapy exposure, IMRT field, and IMRT dose. For patients exposed to chemotherapy, the use of alkylating agents, anthracyclines, and/or epipodophyllotoxins was specified.

\section{Radiotherapy}

IMRT field and dose were evaluated as previously described ${ }^{7}$. Specifically, the IMRT field was categorized by site (e.g., central nervous system [CNS]), head and neck, thorax, abdomen, pelvis, and extremities). If the patient was exposed to IMRT on multiple occasions, the first exposure to IMRT was used for the analysis. If the patient had multiple sites treated with IMRT, the site with the highest dose was selected, and if the prescription dose was the same, both fields were used. If the field treated with IMRT was also exposed to conventional radiotherapy ( $18 \%$ of patients), the total dose was calculated and non-IMRT component was noted, as previously described ${ }^{7}$.

\section{Determination of Second Malignant Neoplasm Development}

Development of SMNs was captured by carefully reviewing the MSKCC medical record, which includes outside correspondence with medical professionals from other treating institutions. Of patients alive at the time of analysis $(\mathrm{n}=284), 70 \%$ and $75 \%$ had follow-up within the last 2 and 3 years, respectively. Of all patients, 200 (61\%) had more than 10 years of follow-up from IMRT initiation. All SMNs were confirmed by biopsy except for one patient whose family opted for comfort care, as previously described ${ }^{7}$. Imaging from the time of SMN development was compared to the original IMRT plan to describe the radiation dose delivered to and isodose level (IDL) at the site of SMN development. Hematologic malignancies following IMRT were recorded separately.

\section{Statistical Analysis}

Survivors were considered at risk of SMN starting 5 years after IMRT. Cumulative incidence estimates of (1) all SMN and (2) second solid malignancies occurring 5 or more years after the date of IMRT initiation were calculated using the cumulative incidence method of competing risks ${ }^{8}$. Sensitivity analyses were also performed to include patients who developed second cancers within 5 years $(n=2)$. Death was considered a competing event within both analyses, with surviving SMN-free patients censored at the date of last 
follow-up. Median time to SMN events is calculated descriptively among patients that experienced an event. Standardized incidence ratios (SIR) were assessed as the ratio of observed/expected cases of second cancer development. Expected rates in the general population were obtained using age-, gender-, and calendar-year specific cancer incidence rates from the Surveillance, Epidemiology, and End Results (SEER) Program ${ }^{9}$. For calendar years after 2018, SEER rates from 2018 were used, which are the most recently available. Excess absolute risk (EAR) was computed by subtracting the expected number of malignancies from the observed number of cases, dividing the difference by the number of person-years of follow-up, and multiplying by 1,000. For the SIR and EAR of second solid cancers, expected rates of solid malignancies were obtained from the SEER Program by excluding malignancies defined as leukemia, and calculations performed as above. Statistical analysis was performed using R $4 \cdot 1 \cdot 0^{10}$.

\section{RESULTS}

\section{Patient Characteristics}

Patient and treatment characteristics are described in Table 1. The median age at IMRT initiation was 9.0 (range, 0.6-21.4) and 90 (28\%) patients were [?]5 years of age. The most common primary cancer was softtissue sarcoma $(n=109,34 \%)$, followed by brain tumor $(n=85,26 \%)$ and neuroblastoma $(n=64,20 \%)$. Most patients received chemotherapy $(\mathrm{n}=282,87 \%)$, many of whom received alkylating agents $(\mathrm{n}=259,80 \%)$. For all patients, the median follow-up was 11.0 years from IMRT (range, 5.1-20.5). Of the 284 patients alive at the time of analysis, median follow-up was 12.0 years from diagnosis (5.4-34.2) and 11.2 years from IMRT initiation (range, 5.1-20.1).

\section{Second Solid Malignancies}

Since the preliminary publication in 2015, 7 additional patients developed a solid SMN for a total of 11 patients. Median time to solid SMN from start of IMRT was 11.0 years (range, 6.8-19.2) among patients with solid SMN, with 10- and 15-year cumulative incidences $1.8 \%$ (95\% CI $0.7-3.9$ ) and $3.5 \%$ (95\% CI 1.4-7.5), respectively (Figure 1). The SIR was 13.7 (95\% CI 6.9-24.6) and EAR was 2.8 per 1,000 personyears (95\% CI 1.0-4.6). Details of the patients who developed a solid SMN are outlined in Table 2. The solid SMNs included high grade glioma $(n=3)$, osteosarcoma $(n=3)$, colon adenocarcinoma $(n=2)$, papillary thyroid cancer $(\mathrm{n}=1)$, widely metastatic cancer of unknown primary $(\mathrm{n}=1)$, and presumed sarcoma $(\mathrm{n}=1)$. Two patients had hereditary retinoblastoma, both of whom developed secondary osteosarcoma. The mean dose to the IMRT field was 53.6 Gy (standard deviation, 24.3). On evaluation of site of SMN in reference to the original IMRT plan as demonstrated in Figure 2, 5 solid SMNs developed within the 100\% IDL of the IMRT field (3 osteosarcomas, 1 presumed sarcoma, 1 high grade glioma), 1 high grade glioma developed within the $80 \%$ IDL of the IMRT boost, 1 high grade glioma developed within the 50\% IDL, and 1 colon adenocarcinoma developed within the $40 \%$ IDL. A colon adenocarcinoma is estimated to have developed within the 70-80\% IDL of the conventional posteroanterior (PA) field as part of craniospinal irradiation at 20.5 years after RT (Figure 3). The papillary thyroid carcinoma was out of the IMRT field. The metastatic cancer of unknown primary could not be categorized in relation to IMRT. The median adjusted prescription dose of IMRT was 54.0 Gy (range, 21.0-96.4) and the median dose to the site of SMN development (excluding the unknown primary case, including the PA field case) was 36.9 Gy (range, 0-96.4).

\section{All Second Malignancies (Solid and Hematologic)}

Since the preliminary publication, 4 additional patients developed a second hematologic malignancy for a total of 6 patients with a second hematologic malignancy. Details of patients who developed a second hematologic malignancy are outlined in Table 3. The median age at IMRT initiation among patients who developed a second hematologic malignancy was 6.7 years (range, 2.9-9.1). Three patients developed myelodysplastic syndrome (MDS), one developed acute myeloid leukemia (AML), and 2 developed MDS/AML. A total of 15 patients developed any SMN (11 solid, 4 hematologic) at a median time of 8.8 years from IMRT initiation (range, 5.9-19.2). Two of the patients who developed hematologic malignancies did so within 5 years following IMRT (AML/MDS at 3.2 years and AML at 3.4 years). The 10- and 15-year cumulative incidences for any SMN 5 years after IMRT were $3.2 \%$ (95\% CI 1.6-5.7) and $4.9 \%$ (95\% CI 2.3-9.0), respectively (Figure 
1). The SIR was 16.2 (95\% CI 9.1-26.8) and EAR was 3.9 per 1000 person-years.

\section{DISCUSSION}

With a median follow-up of more than 10 years, we present the risk of second malignancy after treatment of childhood cancer with IMRT. Since the initial report in 2015, 7 additional patients developed a second solid cancer, nearly all of which occurred more than 10 years after IMRT. This updated analysis with a larger cohort and longer follow-up is important to capture the long-term toxicity of SMN development after IMRT.

The 10-year solid SMN cumulative incidence of $1.8 \%$ reported here falls within the range of risks previously reported following conventional RT and does not suggest a relative increase in SMN from IMRT ${ }^{3,11-15}$. This comparable risk is encouraging given the dose redistribution in IMRT leads to a larger volume of low-dose exposure, which may present a risk for carcinogenesis as a prior report found malignancies developing in tissue receiving less than $2.5 \mathrm{~Gy}^{16}$. Interestingly, in our cohort, second solid malignancies developed most often within the high-dose region, with 5 of 11 subsequent tumors occurring within the $100 \%$ IDL. The lowest doses in our cohort were 15Gy (40\% IDL) and 18Gy (50\% IDL) at the sites where a subsequent colon adenocarcinoma and high-grade glioma developed, respectively. One patient developed a papillary thyroid cancer completely out of the IMRT field. Although thyroid carcinogenesis can occur after exposure of just 0.05Gy, with papillary comprising the most common subtype, the pattern of development here suggests the contribution of other risk factors, such as non-IMRT radiation exposure (e.g. , frequently diagnostic, on-treatment, and/or surveillance imaging) and exposure to alkylating agents, which are known to increase the risk of thyroid cancer more than 2 -fold ${ }^{17,18}$.

In the era of increasing availability of other radiation modalities, such as proton beam RT, it is critical to understand how the risk of second cancers compares between these approaches. While proton beam RT introduces multiple potential advantages, such as sparing "exit" dose and reducing the volume exposed to low- and intermediate dose, it is unclear whether this dosimetric advantage leads to clinically meaningful reduction in development of SMN. On the contrary, it has been theorized that the passive modulation proton technique can potentially expose the patient to an even higher dose of radiation distant from the target compared to IMRT as a function of neutron production from the scattering foil ${ }^{19}$. To address this question, a recent study published reported second cancer risks among 1713 children treated with double-scattered proton therapy with a median of 3.3 years of follow-up ${ }^{20}$. While their study differed from our report by including both benign and malignant tumors, as well as including patients with less than 5 years of follow-up, they do report results on a subset of 549 patients with at least 5 years of follow-up. Among this subset, with a median of 7.1 years of follow-up, the 10-year cumulative incidence of any second solid neoplasm (benign or malignant) was $2.3 \%$, which is less than our preliminary report (3.3\%), leading to their conclusion that proton beam RT does not seem to increase the risk of second cancers and may in fact reduce the risk as seen in adults ${ }^{21,22}$. However, it is important to note that in the current analysis of an expanded cohort with longer follow-up, the 10-year cumulative incidence of second solid malignancies after IMRT is $1.8 \%$. It is difficult to directly compare cumulative risk measurements between studies with different methods and follow-ups as it is possible that the 10-year cumulative incidence of second solid tumors reported by Indelicato et al. may decrease with longer follow-up. Similarly, while the subset of patients with long-term follow-up after proton therapy had a relatively reduced SIR (10 vs 16.2) and EAR (1.2 vs 3.9 per 1,000 person-years) compared to our cohort, these estimates can vary widely with methodology ${ }^{3,13,23}$. These nuances, in addition to the fact that most of the additional subsequent solid tumors developed after 10 years in our cohort, highlight the importance of prolonged follow-up to fully assess risk.

Additional factors beyond radiation therapy can contribute to secondary malignancy risk, including initial tumor histology, genetic predisposition, age, and systemic chemotherapy. In our cohort, all but one patient who developed a second solid tumor had IMRT directed to the head (including brain) and neck, which supports prior data demonstrating an increased risk of second malignancy in patients with CNS tumors ${ }^{3,13,24}$. Five patients had a documented hereditary predisposition syndrome: the 2 patients with hereditary retinoblastoma both developed secondary solid cancers after IMRT, while the 2 patients with Neurofibromatosis Type 1 and one patient with Li Fraumeni Syndrome did not develop a second cancer. Of the 17 patients who 
developed an SMN, all but one had been exposed to chemotherapy, and of those, $94 \%$ received an alkylating agent. Most chemotherapy-related cases of AML/MDS occur within 5 years of treatment regardless of RT exposure $^{25}$; therefore, the 2 hematologic malignancies that developed $<5$ years after treatment in our cohort may be explained by their exposure to epipodophyllotoxins. Younger age is a known risk factor for radiation sensitivity and second cancer development, which was supported by other analyses including the recent study on subsequent neoplasm risk after proton beam $\mathrm{RT}^{20}$. This association was not clear in our data, as $9 \%$ of the 90 patients aged [?] 5 years at IMRT developed a second cancer. Interestingly, of the 6 hematologic malignancies, 2 patients were [?] 5 years of age at time of IMRT, one of whom developed AML/MDS $<5$ years after IMRT.

There are several limitations of this study. Like the preliminary report, this study has a single-institution, retrospective design, but does have a larger sample size of 325 patients and longer median follow-up of 11 years. To ensure adequate follow-up, patients who were lost-to follow-up or died within 5 years of IMRT were excluded. While subsequent malignancies after radiation therapy are typically considered a longer-term risk, reports have shown solid tumors even occurring within 3 years of treatment ${ }^{13,16,20,24}$, and it is possible that these were not captured if the patient died within the 5 -year period. Since our focus was on SMN, benign tumors were not captured, but could also be a separate important treatment-related outcome. Patients who had conventional RT were included and this dose was incorporated into the total calculation of IMRT dose to the field of interest; however, including these patients still provided valuable insight as shown by the patient who developed a second solid cancer within the $80 \%$ IDL of the conventional PA field.

With a longer follow-up of median $>10$ years after IMRT, the 10-year cumulative incidence was reduced to $1.8 \%$; however, the additional solid SMNs nearly all developed 10 years after IMRT, resulting in a 15-year cumulative incidence of $3.5 \%$. While some solid SMNs developed in the lower dose region, the most common site was within the highest dose region, suggesting that the benefit of proton therapy sparing the risk of SMN compared to IMRT may not be profound. Further studies conducted with similar analyses are needed to better compare the risk between these two modalities.

\section{CONFLICTS OF INTEREST}

The authors have no potential conflicts of interest to disclose.

\section{ACKNOWLEDGEMENTS}

None.

\section{REFERNCES}

1. Howlader N, Noone A, Krapcho M, Al E. SEER Cancer Statistics Review, 1975-2018, National Cancer Institute. https://seer.cancer.gov/csr/1975_2018/. Published 2020.

2. Kutanzi K, Lumen A, Koturbash I, Miousse I. Pediatric exposures to ionizing radiation: carcinogenic considerations. Int J Environ Res Public Health . 2016;13:1057.

3. Friedman D, Whitton J, Leisenring W, et al. Subsequent neoplasms in 5-year survivors of childhood cancer: The Childhood Cancer Survivor Study. J Natl Cancer Inst . 2010;102:1083-1095.

4. Kry S, Salehpour M, Followill D, et al. The calculated risk of fatal secondary malignancies from intensitymodulated radiation therapy. Int J Radiat Oncol Biol Phys . 2005;62:1195-1203.

5. Hall J, Wuu S. Radiation induced second cancers: The impact of 3D-CRT and IMRT. Int J Radiat Oncol Biol Phys . 2003;56:83-88.

6. Zelefsky M, Fuks Z, Happersett L, et al. Clinical experience with intensity modulated radiation therapy (IMRT) in prostate cancer.Radiother Oncol . 55AD:241-249.

7. Casey D, Friedman D, Moskowitz C, Hilden P, Al E. Second cancer risk in childhood cancer survivors treated with intensity-modulated radiation therapy (IMRT). Pediatr Blood Cancer . 2015;62(2):311-316. 
8. Aalen O. Nonparametric estimation of partial transition probabilities in multiple decrement models. Ann Stat . 1978;6:534-545.

9. CDC. National Program of Cancer Registries and Surveillance, Epidemiology, and End Results SEER*Stat Database: NPCR and SEER Incidence - U.S. Cancer Statistics Public Use Research Databases (2001-2018). United States Department of Health and Human Services, Ce. https://www.cdc.gov/cancer/uscs/publicuse/. Accessed November 30, 2021.

10. R: A language and environment for statistical computing. https://www.r-project.org/.

11. Jenkinson H, Hawkins M, Stiller C, Winter D, et al. Long-term population based risks of second malignant neoplasms after childhood cancer in Britain. Br J Cancer . 2004;91:1905-1910.

12. Olsen J, Garwicz S, Hertz H, et al. Second malignant neoplasms after cancer in childhood or adolescence. Br Med J . 1993;(307):1030-1036.

13. Inskip P, Curtis R. New malignancies following childhood cancer in the United States. Int J Cancer . 2007;121(10):2233-2240.

14. Mike V, Meadows A, D'Angio G. Incidence of second malignant neoplasms in children: Results of an international study. Lancet . 1982;2:1326-1331.

15. O'Brien M, Donaldson S, Balise R, Whittemore A, Link M. Second malignant neoplasms in survivors of pediatric Hodgkin's lymphoma treated with low-dose radiation and chemotherapy. J Clin Oncol . 2010;28:1232-1239.

16. Diallo I, Haddy N, Adjadj E, et al. Frequency distribution of second solid cancer locations in relation to the irradiated volume among 115 patients treated for childhood cancer. Int J Radiat Oncol Biol Phys . 2009; $74(3): 876-883$.

17. Veiga L, Holmberg E, Anderson H, et al. Thyroid Cancer after Childhood Exposure to External Radiation: An Updated Pooled Analysis of 12 Studies. Radiat Res . 2016;185(5):473-484.

18. Veiga L, Bhatti P, Ronckers C, Sigurdson A, Stovall M, et al. Chemotherapy and thyroid cancer risk: A report from the Childhood Cancer Survivor Study. Epidemiol Biomarkers Prev . 2012;21(1):92-101.

19. Hall E. Intensity-modulated radiation therapy, protons, and the risk of second cancers. Int $J$ Radiat Oncol . 2006;65(1):1-7.

20. Indelicato D, Bates J, Mailhot Vega R, Rotondo R, et al. Second tumor risk in children treated with proton therapy. Pediatr Blood Cancer . 2021;68(7):e28941.

21. Chung C, Yock T, Nelson K, Xu Y, Keating N, Tarbell N. Incidence of second malignancies among patients treated with proton versus photon radiation. Int J Radiat Oncol . 2013;87(1):46-52.

22. Xiang M, Chang D, Pollom E. Second cancer risk after primary cancer treatment with three-dimensional conformal, intensity-modulated, or proton beam radiation therapy. Cancer . 2020;126(15):3560-3568.

23. Garwicz S, Anderson H, Olsen J, et al. Second malignant neoplasms after cancer in childhood and adolescence: a population-based case-control study in the 5 Nordic countries. The Nordic Society of Pediatric Hematology and Oncology. Int J Cancer . 2000;88(4):672-678.

24. Berger C, Trombert-Paviot B, Casagranda L, et al. Second malignant neoplasms following childhood cancer: a study of a recent cohort (1987-2004) from the childhood cancer registry of the Rhone-Alpes region (ARCERRA) in France. Pedatric Heamtol Oncol . 2011;28(5):364-379.

25. Bhatia S. Therapy-related myelodysplasia and acute myeloid leukemia.Semin Oncol . 2013;40(6).

LEGEND 
Figure 1 Cumulative incidence curves for second cancers that developed more than 5 years after IMRT (solid line: second solid cancers; dashed line: any second cancer, including solid and hematologic malignancies).

Figure 2 Two examples of site of solid second malignant neoplasm (SMN) development in reference to IMRT field. A) 11-year-old boy with undifferentiated sarcoma, received $36 \mathrm{~Gy} / 20 \mathrm{fx}$ to the pelvis and para-aortic lymph nodes, developed adenocarcinoma of the cecum 16 years after IMRT at age 27. The site of SMN developed within the $40 \%$ IDL and had received $~ 15 \mathrm{~Gy}$. B) 9-year-old boy with ALL involving the CSF and arachnoid chloroma, received $18 \mathrm{~Gy} / 12 \mathrm{fx}$ to the whole brain with IMRT boost to $24 \mathrm{~Gy} / 16 \mathrm{fx}$, developed glioblastoma of the right parietal lobe 16.5 years after IMRT at age 26. The site of SMN developed within the conventional whole-brain radiotherapy field, within the $80 \%$ IDL of IMRT, and had received a total of 20Gy.

Figure 3 Site of solid second malignant neoplasm (SMN) development in reference to posteroanterior field from craniospinal irradiation as part of treatment for medulloblastoma. This SMN (colon adenocarcinoma) developed 20.5 years after radiation therapy.

\section{Hosted file}

Table 1.docx available at https://authorea.com/users/452578/articles/550606-second-cancerrisk-in-childhood-cancer-survivors-treated-with-intensity-modulated-radiation-therapyimrt-an-updated-analysis-of-more-than-10-years-of-follow-up

\section{Hosted file}

Table 2.docx available at https://authorea.com/users/452578/articles/550606-second-cancerrisk-in-childhood-cancer-survivors-treated-with-intensity-modulated-radiation-therapyimrt-an-updated-analysis-of-more-than-10-years-of-follow-up

\section{Hosted file}

Table 3.docx available at https://authorea.com/users/452578/articles/550606-second-cancerrisk-in-childhood-cancer-survivors-treated-with-intensity-modulated-radiation-therapyimrt-an-updated-analysis-of-more-than-10-years-of-follow-up

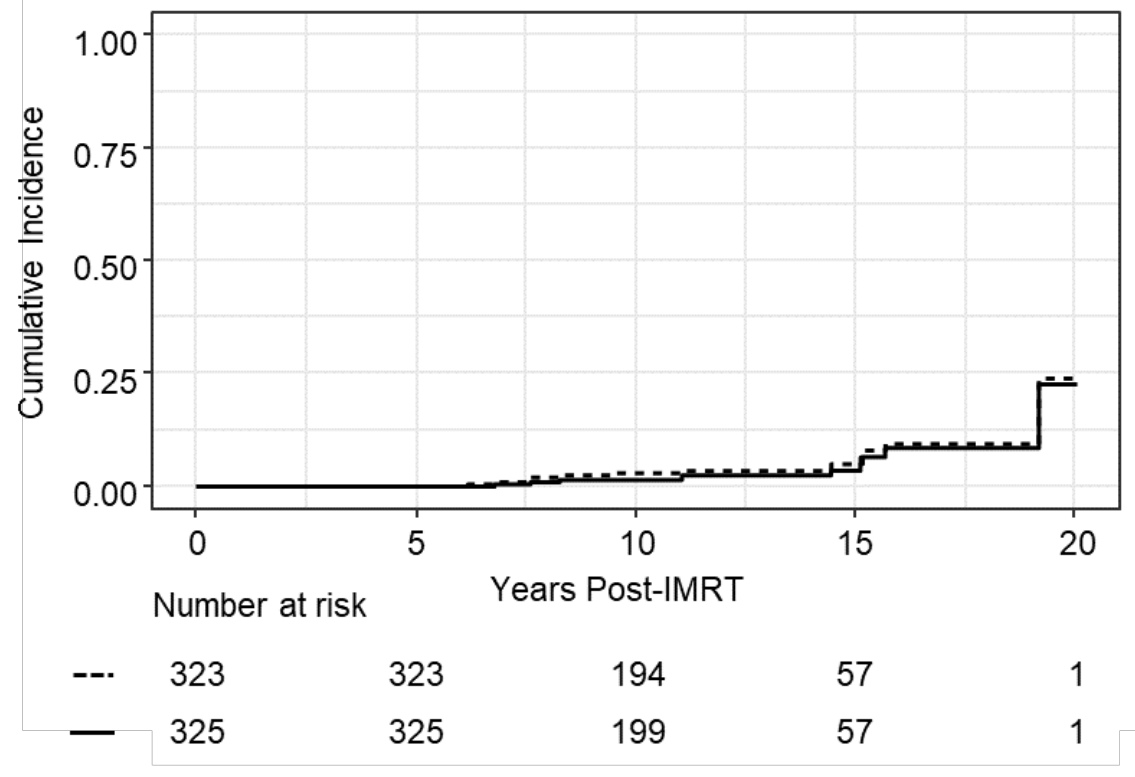

- Second Solid Cancers --. Any SMN 

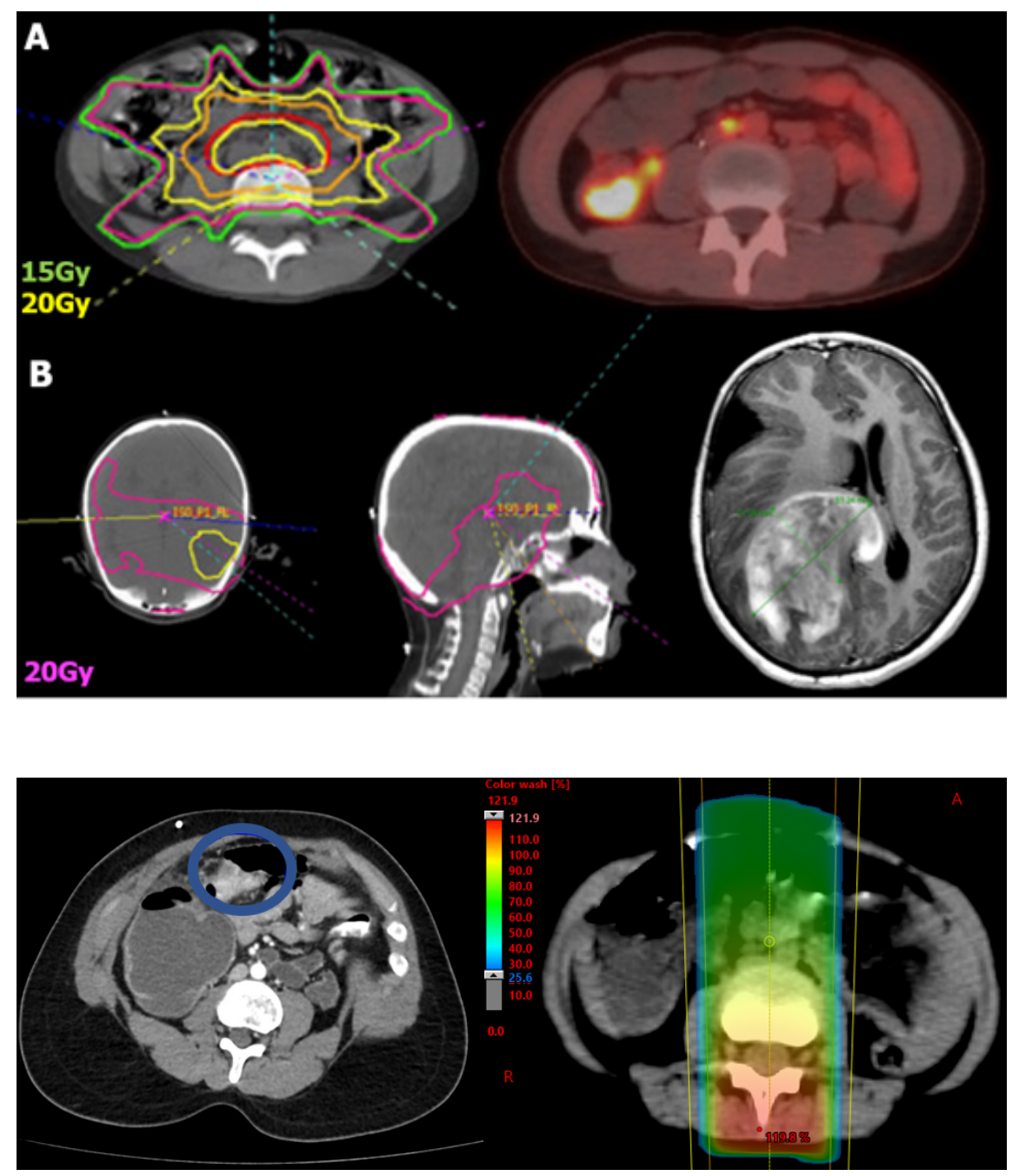